\title{
The Risk of Latent Tuberculosis Infection in Respiratory Therapists in a Country with Intermediate Incidence
}

\author{
Meng-Jer Hsieh, Han-Chung Hu, Kuo-Chin Kao, Hsiu-Ying Cho, Chung-Chi Huang, \\ Ning-Hung Chen, Yen-Li Chou, Chih-Hao Chang, and Ying-Huang Tsai
}

\begin{abstract}
BACKGROUND: Respiratory therapists (RTs) are exposed to aerosols more frequently than other health care workers (HCWs) and might bear a higher risk for tuberculosis (TB) infection. The QuantiFERON-TB Gold (QFTG) test was used to evaluate the risk for TB infection in Taiwan, a country with intermediate TB incidence. METHODS: A cross-sectional screening of HCWs, including RTs and other HCWs, with the QFTG test was conducted in Taiwan between October 2008 and December 2011. Those with initially negative QFTG results accepted repeated QFTG testing 1 y later. The positive rates of QFTG in RTs and other HCWs were compared. The risk factors for positive QFTG and QFTG conversion, including occupational group, age, duration of employment, and gender, were analyzed. RESULTS: A total of 274 HCWs were enrolled, including 43 RTs, 163 nurses, and 68 other HCWs. The positive rates of QFTG were $14.0 \%$ in RTs, $6.1 \%$ in nurses, and $8.8 \%$ in other HCWs, which were not significantly different among the 3 groups. Multivariate analysis demonstrated that the risk for positive QFTG positively correlated with increased age and the duration of employment, but did not relate to gender or occupational group. Of $81 \mathrm{HCWs}$ with initially negative QFTG results, 4 (4.9\%) had positive conversion on repeat QFTG testing 1 y later. The risk for QFTG conversion in HCWs was not related to occupational group, gender, age, or duration of employment. CONCLUSION: RTs had no higher risk for latent TB infection than other HCWs in a country with intermediate TB incidence. Key words: tuberculosis; heath care worker; respiratory therapist; interferon gamma; nosocomial infection. [Respir Care 2019;64(3):313-320. @ 2019 Daedalus Enterprises]
\end{abstract}

\section{Introduction}

There is an increased risk for tuberculosis (TB) infection among health care workers (HCWs) in comparison

\footnotetext{
Drs Hsieh, Chou, and Tsai are affiliated with the Department of Pulmonary and Critical Care Medicine, Chiayi Chang-Gung Memorial Hospital, Chang-Gung Medical Foundation, Taiwan. Drs Hsieh, Hu, Kao, Huang, Chen, and Tsai are affiliated with the Department of Respiratory Therapy, Chang-Gung University, Taiwan. Drs Hu, Kao, Huang, Chen, and Chang are affiliated with the Department of Pulmonary and Critical Care Medicine, Linkou Chang-Gung Memorial Hospital, Chang-Gung Medical Foundation, Taiwan. Drs Hu, Kao, Cho, Huang, and Chen are affiliated with the Department of Respiratory Therapy, Linkou ChangGung Memorial Hospital, Chang-Gung Medical Foundation, Taiwan.
}

The authors disclose a relationship with the Chang-Gung Medical Research Program (No. CMRPG361141).

Dr Hsieh presented a version of this paper at the American Thoracic Society meeting, held May 15-20, 2015, in Denver, Colorado. with the general population, due to the higher risk for occupational exposure to Mycobacterium tuberculosis in the workplace. ${ }^{1-4}$ The risk for transmission depends on different health care settings, occupational groups, local prevalence of $\mathrm{TB}$, and effectiveness of TB infection control measures. ${ }^{5}$ Certain health care workers (HCWs), such as nurses and laboratory workers, have been associated with a higher risk for latent TB infection because of the frequent contact with TB patients or specimens that contained TB bacilli. ${ }^{1,6-9}$ Respiratory therapists (RTs) have contact with aerosols generated by possible TB patients

\footnotetext{
Correspondence: Meng-Jer Hsieh MD, Department of Pulmonary and Critical Care Medicine, Chiayi Chang-Gung Memorial Hospital, ChangGung Medical Foundation. No. 6, West Sec., Jiapu Rd., Puzi City, Chiayi County 613, Taiwan. E-mail: mengjer@cgmh.org.tw.
}

DOI: $10.4187 /$ respcare.06316 
more frequently than other HCWs, which might be associated with a higher risk for TB bacilli exposure. However, the risk for TB infection in RTs has not been reported.

After inhalation of aerosols containing $M$. tuberculosis, the bacilli might be eradicated by the human immune system, result in active TB disease, or become dormant and result in latent TB infection. Latent TB infection is defined as a state of persistent immune response to stimulation by M. tuberculosis antigens without evidence of clinically manifested active TB. ${ }^{10}$ The overall lifetime risk for developing active TB is approximately $10 \%$. Traditionally, latent TB infection was diagnosed using the tuberculin skin test. The Centers for Disease Control and Prevention of the United States approved the use of an interferon $\gamma$-releasing assay (IGRA), QuantiFERON-TB Gold (QFTG), for the diagnosis of latent TB infection in 2005.11 The IGRA had a higher specificity than the tuberculin skin test in persons who had received the bacillus of Calmette and Guérin vaccine. ${ }^{12}$ There has been a routine bacillus of Calmette and Guérin vaccination program for newborns in Taiwan for decades, and most Taiwanese citizens received their bacillus of Calmette and Guérin vaccinations at birth. ${ }^{13,14}$ In this study, we used the QFTG test instead of the tuberculin skin test to diagnose latent TB infection to eliminate the influence of bacillus of Calmette and Guérin vaccination.

Taiwan is a country with intermediate TB burden, although the annual incidence of TB has decreased gradually from $67.4 / 100,000$ people in 2006 to $43.9 / 100,000$ people in 2016. ${ }^{15}$ HCWs are exposed to TB bacilli not only from the community but also from TB patients in the work place. We performed a cross-sectional QFTG testing to assess the risk for latent TB infection in RTs compared to other HCWs, including nurses, physicians, and other members of medical staff in a 3,300-bed tertiary teaching hospital in northern Taiwan. The risk for latent TB infection and QFTG conversion between RTs and other HCWs was compared and analyzed.

\section{Methods}

\section{Study Design and Subjects}

An observational cohort study was conducted from October 2008 to December 2011 among HCWs in LinKou Chang-Gung Memorial Hospital in Taiwan. Our hospital is a 3,300-bed tertiary teaching hospital with 120 RTs. Approximately 900 TB patients were treated in this hospital annually. Hospital employees, including RTs, nurses in chest wards and medical/surgical ICUs, physicians, radiology technicians, and pharmacists $>18 \mathrm{y}$, underwent QFTG testing voluntarily in the period between October 2008 and December 2008. Chest radiographs were taken annually for 3 consecutive years after QFTG testing to rule

\section{QUICK LOOK}

\section{Current knowledge}

Health care workers have an increased risk for tuberculosis infection in comparison with the general population, due to the higher risk for occupational exposure to M. tuberculosis in the workplace.

\section{What this paper contributes to our knowledge}

Respiratory therapists in a country with intermediate tuberculosis incidence had no higher risk for latent tuberculosis infection than other health care workers.

out active pulmonary TB. The annual radiographs in 20092011 were reviewed by 2 chest physicians separately. If there was a difference of opinion in the interpretation, the radiograph was sent for review by the third physician.

This study was conducted in accordance with the amended Declaration of Helsinki. This study was approved by the Institutional Review Board of Chang Gung Memorial Hospital, and written informed consent was obtained from all HCWs.

\section{QuantiFERON-TB Gold Testing}

The QFTG in tube test was performed according to the manufacturer's instructions (Cellestis, Carnegie, Australia). Venous blood was incubated in the Nil control, TB antigen, and phytohemagglutinin (mitogen) control tubes for $16-24 \mathrm{~h}$ at $37^{\circ} \mathrm{C}$. The plasma was harvested from each tube, and measurement of interferon $\gamma$ production in the harvested plasma was measured with enzyme-linked immunosorbent assay. The test was interpreted as positive when the corrected TB antigenstimulated plasma level was $\geq 0.35 \mathrm{IU} / \mathrm{mL}$.

\section{Data Collection}

Baseline data, including age, gender, occupational group, duration of employment, history of TB, status of bacillus of Calmette and Guérin vaccination, history of abnormal chest radiograph, and associated chronic diseases, were recorded. Symptoms of possible active TB were recorded by self-administered questionnaires annually in the 3-y follow-up period.

\section{Statistical Analysis}

Differences between positive and negative QFTG groups were compared with independent $t$ test for continuous data 
RT RISK FOR LATENT TUBERCULOSIS INFECTION

Table 1. Baseline Demographic Data of Health Care Workers Receiving QuantiFERON Tuberculosis Gold Tests

\begin{tabular}{|c|c|c|c|c|c|}
\hline & Nurses & Respiratory Therapists & Other HCWs & Total & $P^{*}$ \\
\hline Age, y & $28.5 \pm 6.0$ & $30.3 \pm 7.4$ & $34.2 \pm 8.2$ & $30.2 \pm 7.2$ & $<.001$ \\
\hline Duration of employment, y & $5.7 \pm 5.8$ & $6.8 \pm 6.6$ & $7.8 \pm 7.9$ & $6.4 \pm 6.4$ & .08 \\
\hline Gender & & & & & $<.001$ \\
\hline Male & 1 & 4 & 35 & 40 & \\
\hline Female & 162 & 39 & 33 & 234 & \\
\hline Age, y & & & & & $<.001$ \\
\hline$\leq 25$ & $57(35.0)$ & $15(34.9)$ & $5(7.4)$ & $77(28.1)$ & \\
\hline $25.1-30$ & $57(35.0)$ & $8(18.6)$ & $28(41.2)$ & $93(33.9)$ & \\
\hline $30.1-35$ & $26(16.0)$ & $12(27.9)$ & $10(14.7)$ & $48(17.5)$ & \\
\hline $35.1-40$ & $16(9.8)$ & $3(7.0)$ & $10(14.7)$ & $29(10.6)$ & \\
\hline$>40$ & $7(4.3)$ & $5(11.6)$ & $15(22.1)$ & $27(9.9)$ & \\
\hline \multicolumn{5}{|l|}{ Duration of employment, $y$} & .002 \\
\hline$\leq 1$ & $49(30.1)$ & $13(30.2)$ & $6(8.8)$ & $68(24.8)$ & \\
\hline $1.1-5$ & $49(30.1)$ & $9(20.9)$ & $32(47.1)$ & $90(32.8)$ & \\
\hline $5.1-10$ & $32(19.6)$ & $12(27.9)$ & $10(14.7)$ & $54(19.7)$ & \\
\hline $11.1-15$ & $22(13.5)$ & $5(11.6)$ & $8(11.8)$ & $35(12.8)$ & \\
\hline$>15$ & $11(6.7)$ & $4(9.3)$ & $12(17.6)$ & $27(9.9)$ & \\
\hline \multicolumn{6}{|c|}{$\begin{array}{l}\text { Data presented as mean } \pm \text { SD or } n(\%) \text {. } \\
* \text { Compared with analysis of variance for interval data or chi square test for categorical data. } \\
\mathrm{HCW}=\text { health care worker }\end{array}$} \\
\hline
\end{tabular}

or with chi square or Fisher exact tests (when the expected number of events was $<5$ ) for categorical data. Multiple logistic regressions were used, with positive or negative QFTG as the dependent variable and age, gender, occupational group, and duration of employment as independent variables. Age and duration of employment were grouped as categorical data and entered into the regression separately due to the high correlation between them. Likewise, gender was not used for the multiple logistic regressions due to the high correlation with the occupational groups. The statistical analysis was performed using SPSS (SPSS for Windows, v.13.0; SPSS, Chicago, Illinois); the significance level $(\alpha)$ for all statistical tests was set at 0.05 , and $P<.05$ was considered statistically significant.

\section{Results}

A total of $274 \mathrm{HCWs}$ were enrolled voluntarily, including 43 RTs, 163 nurses, and 68 other HCWs, including physicians, pharmacists, and technicians in the department of radiology and some other administrative staff in the wards. Among 163 nurses enrolled in this study, 61 were from the chest ward, 20 were from the general internal medicine ward, 17 were from the respiratory care center, 28 were from the medical ICU, and 37 were from the neurosurgery ICU. Demographic data are shown in Table 1 . The nurses and RTs were significantly younger than the third group, which included other HCWs. There was no statistical difference in the mean duration of employ- ment among the 3 occupational groups. The age and duration of employment of the HCWs were divided into 5 groups each, and there were significant differences in the distribution of age $(P<.001)$ and duration of employment $(P=.002)$ among the 3 occupational groups.

\section{Baseline QFTG}

The overall positive rate of QFTG in all HCWs in the first year was $8.0 \%$. The rates of positive QFTG were $14.0 \%$ in RTs, $6.1 \%$ in nurses, and $8.8 \%$ in other HCWs, respectively, which did not differ significantly among the 3 groups. As shown in Table 2, HCWs with positive QFTG were significantly older $(35.0 \pm 7.6$ vs $29.8 \pm 7.1 \mathrm{y}$, $P<.001)$ and had longer duration of employment $(11.1 \pm 8.1$ vs $6.0 \pm 6.1 \mathrm{y}, P<.001)$ than those with negative QFTG. Table 3 presents the results of multivariate logistic regression analysis, which demonstrates that the risk for positive QFTG was not related to gender or occupational groups. The odds ratios of positive QFTG increased gradually with increased age and duration of employment. The risk for positive QFTG was significantly higher in HCWs $>40 \mathrm{y}$ old or with a duration of employment $>10 \mathrm{y}$. The risk for positive QFTG was 7.49 times higher in HCWs $>40 \mathrm{y}$ old than in those younger than $25 \mathrm{y}$. Meanwhile, in comparison with the HCWs with duration of employment $<1 \mathrm{y}$, the risks for positive QFTG were 5.84 and 9.84 times higher in HCWs with a duration of employment of 10.1-15 y and >15 y, respectively. 
Table 2. Baseline QuantiFERON Tuberculosis Gold Tests in Different Occupational Groups

\begin{tabular}{|c|c|c|c|c|c|}
\hline & \multirow{2}{*}{ Total } & \multicolumn{3}{|c|}{ QuantiFERON Tuberculosis Gold Result } & \multirow{2}{*}{$P^{*}$} \\
\hline & & Positive & Negative & Positive Rate, $\%$ & \\
\hline Number, $n$ & 274 & 22 & 252 & 8.0 & \\
\hline \multicolumn{6}{|l|}{ Gender, $n(\%)$} \\
\hline Male & $40(14.6)$ & $3(13.6)$ & $37(14.7)$ & 7.5 & \multirow[t]{2}{*}{.89} \\
\hline Female & $234(85.4)$ & $19(86.4)$ & $215(85.3)$ & 8.1 & \\
\hline \multicolumn{5}{|l|}{ Occupational group, $n(\%)$} & \multirow[t]{4}{*}{.25} \\
\hline Nurses & $163(59.5)$ & $10(45.5)$ & $153(60.7)$ & 6.1 & \\
\hline Respiratory therapists & $43(15.7)$ & $6(27.3)$ & $37(14.7)$ & 14.0 & \\
\hline Other health care workers & $68(24.8)$ & $6(27.3)$ & $62(24.6)$ & 8.8 & \\
\hline Age, y & $30.2 \pm 7.2$ & $35.0 \pm 7.6$ & $29.8 \pm 7.1$ & & $<.001$ \\
\hline$\leq 25$ & $77(28.1)$ & $3(13.6)$ & $74(29.4)$ & 3.9 & \multirow[t]{5}{*}{.02} \\
\hline $25.1-30$ & $93(33.9)$ & $4(18.2)$ & $89(35.3)$ & 4.3 & \\
\hline $30.1-35$ & $48(17.5)$ & $6(27.3)$ & $42(16.7)$ & 12.5 & \\
\hline $35.1-40$ & $29(10.6)$ & $3(13.6)$ & $26(10.3)$ & 10.3 & \\
\hline$>40$ & $27(9.9)$ & $6(27.3)$ & $21(8.3)$ & 22.2 & \\
\hline \multirow{6}{*}{$\begin{array}{l}\text { Duration of employment, y } \\
\quad \leq 1 \\
1.1-5 \\
5.1-10 \\
10.1-15 \\
>15\end{array}$} & $6.4 \pm 6.4$ & $11.1 \pm 8.1$ & $6.0 \pm 6.1$ & & \multirow{6}{*}{$\begin{array}{l}.001 \\
.02\end{array}$} \\
\hline & $68(24.8)$ & $2(9.1)$ & $66(26.2 \%)$ & 2.9 & \\
\hline & $90(2.8)$ & $5(22.7)$ & $85(33.7)$ & 5.6 & \\
\hline & 54 (19.7) & $4(18.2)$ & $50(19.8)$ & 7.4 & \\
\hline & $35(12.8)$ & $5(22.7)$ & $30(11.9)$ & 14.3 & \\
\hline & 27 (9.9) & $6(27.3)$ & $21(8.3)$ & 22.2 & \\
\hline
\end{tabular}

\section{Follow-up QFTG}

The results of repeated QFTG testing in the next year are shown in Table 4. Eighty-one of the $252 \mathrm{HCWs}$ with negative QFTG in the first year repeated their QFTG in the next year, and only $4(4.9 \%)$ had positive conversion. The percentage of QFTG conversion was not related to occupational group, gender, age, or the duration of employment. The numbers of HCWs enrolled in the study and the results of QFTG are shown in Figure 1.

\section{Chest Radiograph Screening}

None of the subjects in this study had radiographic evidence of active pulmonary TB during the 3-year follow-up.

\section{Discussion}

The risk for TB infection in RTs has not been reported before, although the risk might be reasonably higher than for other HCWs due to occupational aerosol exposure. Our study is the first to demonstrate that RTs had no higher risk for positive QFTG testing (ie, latent TB infection) than other HCWs. Multiple logistic regression analyses showed that the rates of positive QFTG in HCWs were positively correlated with increased age and the duration of employment, but they were not related to gender or occupational group. With a low number of HCWs receiving repeat QFTG, there was no statistically significant difference in the rates of QFTG conversion among HCWs across different occupational groups, gender, age groups, or duration of employment, which probably indicated that the risk for recent TB infection was not related to these factors in this hospital. However, the evidence is weak with the limited sample size.

In the United Kingdom, a country with lower TB incidence, a higher percentage of TB cases occurred in HCWs born abroad. After stratifying by country of birth, there was no increased TB incidence in HCWs. ${ }^{16}$ Nevertheless, in countries with higher TB incidence, such as Rwanda and South Africa, HCWs do have increased risk for latent TB infection. ${ }^{17,18}$ An adjusted odds ratio of a positive tuberculin skin test was 2.71 times greater among $\mathrm{HCWs}$ than among school workers in Rwanda. ${ }^{17}$ South African HCWs experienced a TB incidence rate double that of the general population. ${ }^{19}$ The higher risk for latent TB infection in countries with high TB incidences might arise from the higher chance of exposure to TB patients in their workplace. Taiwan is a country with intermediate TB incidence, and there could be a higher risk for TB infection in HCWs. ${ }^{20}$ Because we lacked a non-HCW control, the risk for latent TB infection between HCWs and non-HCW in- 
Table 3. Logistic Regression Analysis of the Risk Factors for Positive QuantiFERON Tuberculosis Gold Tests

\begin{tabular}{|c|c|c|c|c|c|c|c|}
\hline & \multirow{2}{*}{$\begin{array}{c}\text { Positive } \\
\text { QuantiFERON } \\
\text { Tuberculosis } \\
\text { Gold/Total, } n(\%)\end{array}$} & \multicolumn{2}{|l|}{ Unadjusted } & \multicolumn{2}{|c|}{ Adjusted Model 1} & \multicolumn{2}{|c|}{ Adjusted Model 2} \\
\hline & & OR $(95 \% \mathrm{CI})$ & $P$ & OR $(95 \% \mathrm{CI})$ & $P$ & OR $(95 \% \mathrm{CI})$ & $P$ \\
\hline \multicolumn{8}{|l|}{ Gender } \\
\hline Male & $3 / 40(7.5)$ & $1.0(\mathrm{NA})$ & NA & & & & \\
\hline Female & $19 / 234(8.1)$ & $0.89(0.31-3.87)$ & .81 & & & & \\
\hline \multicolumn{8}{|l|}{ Occupational group } \\
\hline Nurses & $10 / 163(6.1)$ & 1.0 (NA) & NA & $1.0(\mathrm{NA})$ & NA & $1.0(\mathrm{NA})$ & NA \\
\hline Respiratory therapists & $6 / 43(14.0)$ & $2.48(0.85-7.26)$ & .10 & $2.48(0.81-7.57)$ & .11 & $1.94(0.63-5.97)$ & .25 \\
\hline Other health care workers & $6 / 68(8.8)$ & $1.48(0.52-4.25)$ & .47 & $1.12(0.37-3.38)$ & .85 & $0.92(0.29-2.90)$ & .89 \\
\hline \multicolumn{8}{|l|}{ Age, y } \\
\hline$\leq 25$ & 3/77 (2.9) & 1.0 (NA) & NA & & & 1.0 (NA) & NA \\
\hline $25.1-30$ & $4 / 93(5.6)$ & $1.44(0.27-7.66)$ & .67 & & & $1.23(0.26-5.82)$ & .79 \\
\hline $30.1-35$ & $6 / 48(7.4)$ & $4.19(0.81-21.74)$ & .09 & & & $3.44(0.81-14.68)$ & .95 \\
\hline $35.1-40$ & $3 / 29(14.3)$ & $3.21(0.51-20.33)$ & .22 & & & $3.14(0.58-17.00)$ & .18 \\
\hline$>40$ & $6 / 27(22.2)$ & $11.67(2.22-61.20)$ & .004 & & & $7.49(1.58-35.57)$ & .01 \\
\hline \multicolumn{8}{|l|}{ Duration of employment, $y$} \\
\hline$\leq 1$ & 2/68 (2.9) & 1.0 (NA) & NA & 1.0 (NA) & NA & & \\
\hline $1.1-5$ & $5 / 90(5.6)$ & $1.94(0.37-10.32)$ & .44 & $2.11(0.39-11.57)$ & .39 & & \\
\hline $5.1-10$ & 4/54 (7.4) & $2.64(0.47-15.00)$ & .27 & $2.55(0.44-14.63)$ & .29 & & \\
\hline $10.1-15$ & $5 / 35(14.3)$ & $5.50(1.01-29.98)$ & .049 & $5.84(1.05-32.37)$ & .040 & & \\
\hline$>15$ & $6 / 27(9.9)$ & $9.42(1.77-50.28)$ & .009 & $9.84(1.76-55.10)$ & .009 & & \\
\hline $\begin{array}{l}\text { For adjusted model } 1, \text { Nagelkerke's } \mathrm{R}^{2} \\
\mathrm{OR}=\text { odds ratio } \\
\mathrm{CI}=\text { confidence interval } \\
\mathrm{NA}=\text { not applicable }\end{array}$ & $.109, P=.041$; for adjuste & odel 2, Nagelkerke's $R^{2}=0$ & $P=.0$ & & & & \\
\hline
\end{tabular}

dividuals could not be compared in this study. However, the risk for latent TB infection in HCWs in different occupational groups was compared in this study, which showed that the risks of latent TB infection were similar among HCWs in different departments.

With regard to the risk factors for latent TB infection in HCWs, multiple logistic regression analysis revealed that the rates of positive QFTG results increased with age and duration of employment but were not related to gender or occupational group. The rate of positive IGRA has been shown to increase with age in close contacts ${ }^{12}$ and in HCWs. ${ }^{21}$ The increased rate of positive QFTG results with increased age and duration of employment indicate that the risk for latent TB infection is related to the accumulated exposure to TB bacilli in the commonly shared environment but was not related to specific working departments. The absence of a significant difference in the rates of QFTG conversion between RT and non-RT populations was also proven with the repeated QFTG testing. The overall rate of IGRA conversion was $4.9 \%$ in this study, which was numerically higher than the rate in the United States, ${ }^{7,22}$ which is probably related to the higher TB incidence in Taiwan. The risk for exogenous infection increased with increased TB incidence in different countries. ${ }^{23}$ For the HCWs in Taiwan, a country with intermediate TB incidence, the risk for TB infection arises not only from their working environment, but also from the communities in which they live.

RTs can be exposed to aerosols that contain infectious materials such as TB bacilli. The possible protective factors from occupational TB infections include sufficient knowledge about TB, clinician experience in interpreting chest radiographs, occupational alertness, and proper personal protection. The RTs in our hospital have regular continuing education not only about respiratory therapy but also about TB disease and chest radiograph reading. They are familiar with clinical and radiological presentations of pulmonary TB so that they can be more aware of patients with possible TB disease. In addition, RTs wear N95 respirators routinely in the ICU, which protect them from inhaling possibly contagious aerosols.

We did not compare the results of QFTG with tuberculin skin tests in this study because it was evident that there was disagreement between the tuberculin skin test and IGRA in people vaccinated with bacillus of Calmette and Guérin. ${ }^{12}$ A cross-sectional study conducted by Hung and co-workers ${ }^{20}$ demonstrated that the positive rates of tuberculin skin test and QFTG in HCWs vaccinated with bacillus of Calmette and Guérin were $88.8 \%$ and $14.5 \%$, respectively. The agreement between these 2 tests was 
Table 4. Analysis of Repeated QuantiFERON Tuberculosis Gold Tests in Health Care Workers with Initial Negative Tests

\begin{tabular}{|c|c|c|c|c|c|}
\hline & \multirow{2}{*}{ Total } & \multicolumn{3}{|c|}{ QuantiFERON Tuberculosis Gold Result } & \multirow{2}{*}{$P^{*}$} \\
\hline & & Positive & Negative & Positive Rate, \% & \\
\hline Number, $n$ & 81 & 4 & 77 & 4.9 & \\
\hline \multicolumn{5}{|l|}{ Gender } & $>.99$ \\
\hline Male & $4(4.9)$ & $0(0)$ & $4(5.2)$ & 0 & \\
\hline Female & $77(95.1)$ & $4(100)$ & $73(94.8)$ & 5.2 & \\
\hline \multicolumn{5}{|l|}{ Occupational group (1) } & $>.99$ \\
\hline Nurses & $62(76.5)$ & $3(75.0)$ & $59(76.6)$ & 4.8 & \\
\hline Respiratory therapists & $16(19.8)$ & $1(25.0)$ & $15(19.5)$ & 6.3 & \\
\hline Other health care workers & $3(3.7)$ & $0(0)$ & $3(3.9)$ & 0 & \\
\hline \multicolumn{5}{|l|}{ Occupational group (2) } & $>.99$ \\
\hline Respiratory therapists & $15(18.5)$ & $1(25.9)$ & $15(19.5)$ & 6.3 & \\
\hline Other health care workers & $62(81.5)$ & $3(75.0)$ & $62(80.5)$ & 4.6 & \\
\hline \multicolumn{5}{|l|}{ Age, y } & .93 \\
\hline$\leq 25$ & $29(35.8)$ & $2(50.0)$ & $27(35.1)$ & 6.9 & \\
\hline $25.1-30$ & $25(30.9)$ & $2(50.0)$ & $23(29.9)$ & 8.0 & \\
\hline $30.1-35$ & $14(17.3)$ & $0(0)$ & $14(18.2)$ & 0 & \\
\hline $35.1-40$ & $7(8.6)$ & $0(0)$ & $7(9.1)$ & 0 & \\
\hline$>40$ & $6(7.4)$ & $0(0)$ & $6(7.8)$ & 0 & \\
\hline \multicolumn{5}{|l|}{ Duration of employment, y } & .84 \\
\hline$\leq 1$ & $24(29.6)$ & $2(50.0)$ & $22(28.6)$ & 8.3 & \\
\hline $1.1-5$ & $27(33.3)$ & $2(50.0)$ & $25(32.5)$ & 7.4 & \\
\hline $5.1-10$ & $17(21.0)$ & $0(0)$ & $17(22.1)$ & 0 & \\
\hline $10.1-15$ & $7(8.6)$ & $0(0)$ & $7(9.1)$ & 0 & \\
\hline$>15$ & $6(7.4)$ & $0(0)$ & $7(9.1)$ & 0 & \\
\hline
\end{tabular}

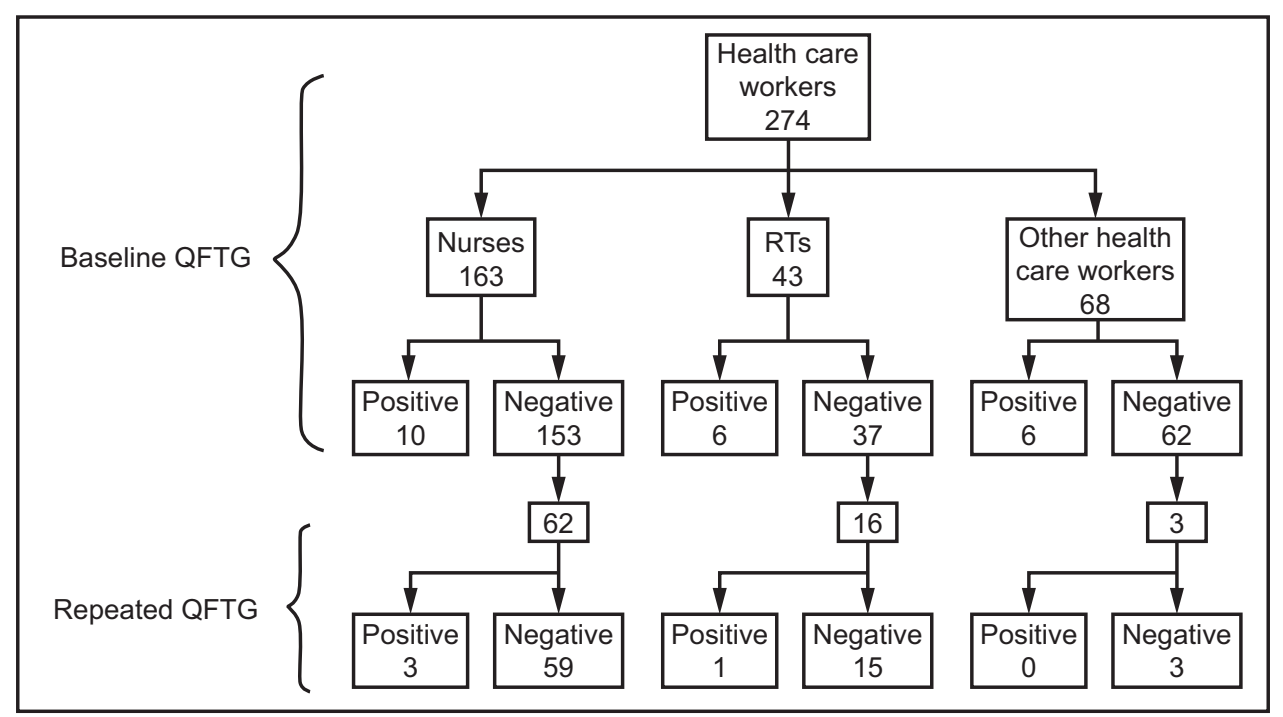

Fig. 1. Flow chart and QTFG results. The repeated test was the following year. QTFG $=$ QuantiFERON Tuberculosis Gold test; RT $=$ respiratory therapist.

poor. The National Immunization Program has included neonatal bacillus of Calmette and Guérin vaccination since 1965, and most Taiwanese citizens have been vaccinated with bacillus of Calmette and Guérin. The coverage rate has remained at $97 \%$ since $2001 .^{13,14}$ This nationwide bacillus of Calmette and Guérin vaccination program might 
affect the positive rate of tuberculin skin test in Taiwan, and therefore interferon $\gamma$-release assays (IRGA) would be the appropriate tests to evaluate latent TB infection in Taiwan.

There are some limitations in this study. First, there was no non-HCW control, so we could not compare the risks of latent TB infection between HCWs and the general population. Second, this is a prospective cohort study instead of a randomized, controlled study, so the age and seniority distribution among different professional disciplines was not matched. For this reason, we performed 2 models of logistic regression analysis using either age or duration of employment, along with gender and the working groups as independent factors to evaluate the risk for positive QFTG in HCWs. Third, RTs make up a specific group of medical staff, and the number of RTs is relatively small in a single hospital. The sample size of RTs is markedly limited in this single-center study, even in this 3,300-bed tertiary teaching hospital. Finally, the number of HCWs who received the repeat QFTG was further limited by the initial number of HCWs enrolled and the willingness of QFTG-negative HCWs to be tested repeatedly because they thought they were not infected. Due to the limited number of HCWs with repeated QFTG the following year, the number of QFTG conversions is relatively low and might not reach statistical significance. There might have been a stronger statistical power if we had more participants for annual IGRA testing to evaluate the risk for recent TB infection by QFTG conversion; however, the number of HCWs needed for a better statistical power could be too large to be achieved.

\section{Conclusions}

RTs in a tertiary teaching hospital in Taiwan, a country with intermediate TB incidence, had no higher risk for latent TB infection than other HCWs. The rates of positive QFTG increased with age and the duration of employment but were not related to their gender or occupational groups. The positive QFTG conversion rates (ie, risk for recent TB infection) were similar in different occupational groups, gender, age, and duration of employment. The risks of latent $\mathrm{TB}$ infection to RTs and other HCWs might be related to exposure to TB bacilli in the commonly shared environment, including the community and the hospital. The importance of continuing education for the recognition of pulmonary $\mathrm{TB}$ and adequate personal protection against TB infection should be emphasized to decrease the risk of TB transmission in health care facilities, especially in countries with intermediate or high TB burden.

\section{REFERENCES}

1. Rafiza S, Rampal KG. Serial testing of Malaysian health care workers with QuantiFERON-TB Gold In-Tube. Int J Tuberc Lung Dis 2012;16(2):163-168.
2. Centers for Disease Control and Prevention, USA. Guidelines for the investigation of contacts of persons with infectious tuberculosis: recommendations from the National Tuberculosis Controllers Association and CDC. MMWR Recomm Rep 2005;54(RR-15):1-37.

3. Menzies D, Fanning A, Yuan L, Fitzgerald M. Tuberculosis among health care workers. N Engl J Med 1995;332(2):92-98.

4. Baussano I, Nunn P, Williams B, Pivetta E, Bugiani M, Scano F. Tuberculosis among health care workers. Emerg Infect Dis 2011; 17(3):488-494.

5. Menzies D, Joshi R, Pai M. Risk of tuberculosis infection and disease associated with work in health care settings. Int $\mathrm{J}$ Tuberc Lung Dis 2007;11(6):593-605.

6. Menzies D, Fanning A, Yuan L, FitzGerald JM, Canadian Collaborative Group in Nosocomial Transmission of Tuberculosis. Factors associated with tuberculin conversion in Canadian microbiology and pathology workers. Am J Respir Crit Care Med 2003;167(4):599-602.

7. Fong KS, Tomford JW, Teixeira L, Fraser TG, van Duin D, YenLieberman B, et al. Challenges of interferon- release assay conversions in serial testing of health-care workers in a TB control program. Chest 2012;142(1):55-62.

8. Boudreau AY, Baron SL, Steenland NK, Van Gilder TJ, Decker JA, Galson SK, et al. Occupational risk of Mycobacterium tuberculosis infection in hospital workers. Am J Ind Med 1997;32(5):528-534.

9. Babus V. Tuberculosis morbidity risk in medical nurses in specialized institutions for the treatment of lung diseases in Zagreb. Int $\mathrm{J}$ Tuberc Lung Dis 1997;1(3):254-258.

10. World Health Organization. Latent TB Infection : Updated and consolidated guidelines for programmatic management, 2018. http:// www.who.int/tb/publications/2018/latent-tuberculosis-infection/en. Accessed September 5, 2018.

11. Mazurek GH, Jereb J, Lobue P, Iademarco MF, Metchock B, Vernon A, et al. Guidelines for using the QuantiFERON-TB Gold test for detecting Mycobacterium tuberculosis infection, United States. MMWR Morb Mortal Wkly Rep 2005;54(RR-15):49-55 [Erratum appears in MMWR Morb Mortal Wkly Rep 2005;54(50):1288].

12. Diel R, Loddenkemper R, Meywald-Walter K, Niemann S, Nienhaus A, Diel R, et al. Predictive value of a whole blood IFN-gamma assay for the development of active tuberculosis disease after recent infection with Mycobacterium tuberculosis. Am J Respir Crit Care Med 2008;177(10):1164-1170.

13. Jou R, Huang WL, Su WJ. Tokyo-172 BCG vaccination complications, Taiwan. Emerg Infect Dis 2009;15(9):1525-1526.

14. Chan PC, Chang LY, Wu YC, Lu CY, Kuo HS, Lee CY, et al. Age-specific cut-offs for the tuberculin skin test to detect latent tuberculosis in BCG-vaccinated children. Int J Tuberc Lung Dis 2008;12(12):1401-1406.

15. Center for Disease Control, Taiwan, ROC. Annual TB incidence from 2005 to 2016, Taiwan. http://daily.cdc.gov.tw/stoptb/CareMagChart. aspx $?$ treeid $=3 \mathrm{f} 2310 \mathrm{~b} 85436188 \mathrm{~d} \&$ nowtreeid $=$ D3A2CA330934BE69. Accessed January 3, 2018.

16. Davidson JA, Lalor MK, Anderson LF, Tamne S, Abubakar I, Thomas HL. TB in healthcare workers in the UK: a cohort analysis 20092013. Thorax 2017;72(7):654-659.

17. Rutanga C, Lowrance DW, Oeltmann JE, Mutembayire G, Willis M, Uwizeye CB, et al. Latent tuberculosis infection and associated factors among health care workers in Kigali, Rwanda. PLoS ONE 2015; 10(4):e0124485.

18. McCarthy KM, Scott LE, Gous N, Tellie M, Venter WD, Stevens WS, et al. High incidence of latent tuberculous infection among South African health workers: an urgent call for action. Int J Tuberc Lung Dis 2015;19(6):647-653.

19. Claassens MM, van Schalkwyk C, du Toit E, Roest E, Lombard CJ, Enarson DA, et al. Tuberculosis in healthcare workers and infection 


\section{RT RISK FOR LATENT TUBERCULOSIS INFECTION}

control measures at primary healthcare facilities in South Africa. PLoS ONE 2013;8(10):e76272.

20. Hung WT, Lee SS, Sy CL, Wu KS, Chen JK, Tsai HC, et al. Prevalence of latent tuberculosis infection in BCG-vaccinated healthcare workers by using an interferon-gamma release assay and the tuberculin skin test in an intermediate tuberculosis burden country. J Microbiol Immunol Infect 2015;48(2):147-152.

21. Nienhaus A, Gariepy PK, Trouve C, Lhaumet C, Toureau J, Peters C. Tuberculosis screening at the Sainte-Anne Hospital in
Paris: results of first and second IGRA. J Occup Med Toxicol 2014;9:24

22. Joshi M, Monson TP, Joshi A, Woods GL. IFN-gamma release assay conversions and reversions. Challenges with serial testing in U.S. health care workers. Ann Am Thorac Soc 2014;11(3):296-302.

23. Uys PW, van Helden PD, Hargrove JW. Tuberculosis reinfection rate as a proportion of total infection rate correlates with the logarithm of the incidence rate: a mathematical model. J R Soc Interface 2009;6(30):11-15. 